\title{
Interpositional carotid artery bypass strategies in the surgical management of aneurysms and tumors of the skull base
}

\author{
James K. LiU, M.D., and William T. Couldwell, M.D., Ph.D. \\ Department of Neurosurgery, University of Utah School of Medicine, Salt Lake City, Utah
}

\begin{abstract}
Cerebral revascularization is an important component in the surgical management of complex skull base tumors and aneurysms. Patients who harbor complex aneurysms that cannot be clipped directly and in whom parent vessel occlusion cannot be tolerated may require cerebrovascular bypass surgery. In cases in which skull base tumors encase the carotid artery (CA) and a resection is desired, a cerebrovascular bypass may be necessary in planned CA occlusion or sacrifice. In this review the authors discuss options for performing high-flow anterograde interposition CA bypass for lesions of the skull base. The authors review three important bypass techniques involving saphenous vein grafts: the cervical-to-petrous internal carotid artery (ICA), petrous-to-supraclinoid ICA, and cervical-to-supraclinoid ICA bypass. These revascularization techniques are important tools in the surgical treatment of complex aneurysms and tumors of the skull base and cavernous sinus.
\end{abstract}

\section{KeY WoRdS - interpositional vein bypass - skull base tumor • aneurysm • cerebral revascularization}

Cerebrovascular bypass procedures remain important strategies in the surgical management of complex aneurysms and skull base tumors. Giant and fusiform aneurysms not amenable to direct clipping may require parent vessel occlusion and a bypass surgery. ${ }^{4,6,10-12,16}$ Skull base tumors involving the ICA may require sacrifice of the ICA if a resection is desired, especially in cases involving malignant tumors. ${ }^{8,10,13,14}$

When acute sacrifice of the ICA is necessary, high-flow revascularization may be indicated, either to restore adequate collateral flow in a patient with insufficient cerebrovascular reserve or to preserve cerebrovascular reserve in a young patient with long life expectancy. Although most patients remain asymptomatic after ligation of the ICA, hypoperfusion in the affected hemisphere may occur in some patients who are predisposed to a high risk of ischemic complications. ${ }^{9}$ False-negative results demonstrated on balloon occlusion tests fail to identify those at risk of ischemic complications after sacrifice of the ICA. ${ }^{7,15}$ This has prompted some authors to recommend universal cerebrovascular bypass whenever the ICA is sacrificed to avoid an ischemic stroke. ${ }^{6}$ Because the controversy surrounding the relative and absolute indications

Abbreviations used in this paper: $\mathrm{CA}=$ carotid artery; $\mathrm{CT}=$ computerized tomography; ICA = internal CA; OA = ophthalmic artery; $\mathrm{SVG}=$ saphenous vein graft. for performing bypass when planning CA ligation is well recognized, the goal of this review is to discuss the operative strategies and different options for performing an interpositional high-flow bypass.

Reconstruction of the ICA with the use of interpositional SVGs for the management of giant aneurysms was popularized by Sundt and colleagues in the early 1980s. ${ }^{4,18-20}$ Ideally, the interpositional graft should be as short as possible and should provide blood flow that approximates that of the bypassed ICA. End-to-end anastomosis at both extremes of the graft is preferred to reduce turbulent blood flow and promote graft patency. ${ }^{16}$ The choice of bypass conduits (arterial compared with venous) is reviewed in another article in this issue of Neurosurgical Focus. We focus here on high-flow interpositional bypass techniques used to treat aneurysms and skull base tumors involving the high cervical, petrous, and cavernous sinus CA. We review specifically the cervical-to-petrous ICA, petrous-tointradural ICA, and cervical-to-intradural ICA bypass procedures. ${ }^{1,3,8,17}$

\section{INTERPOSITIONAL CAROTID ARTERY BYPASS TECHNIQUES}

\section{Cervical-to-Petrous ICA Bypass}

The cervical-to-petrous ICA bypass is a useful intervention in the management of high cervical or skull base 
lesions, including tumors or dissecting or saccular aneurysms. Miyazaki, et al., ${ }^{8}$ undertook a cervical-to-petrous ICA saphenous vein interposition bypass in two patients after radical resection of high-cervical paragangliomas involving the ICA. Fitzpatrick, et al., ${ }^{3}$ performed a similar bypass technique to treat three patients with high cervical ICA dissection lesions refractory to maximal medical therapy (Fig. 1).

The petrous CA can be readily exposed through a temporal craniotomy via a subtemporal extradural approach. ${ }^{5}$ A temporal craniotomy is performed in the same fashion as a standard subtemporal approach. The squamous portion of the temporal bone and the superior/posterior portion of the zygomatic arch are drilled to allow a flat approach to the floor of the temporal fossa. The important anatomical landmarks are the foramen spinosum and the foramen ovale, which are identified extradurally. Brain relaxation is facilitated by administration of intravenous mannitol and, occasionally, placement of a lumbar drain to minimize temporal lobe retraction. After division of the middle meningeal artery, a thin layer of bone posterior to the third division of the trigeminal nerve and medial to the foramen spinosum is drilled away to expose a $1-\mathrm{cm}$ length of the horizontal segment of the petrous ICA suitable for grafting. ${ }^{8}$ Exposure of the $\mathrm{CA}$ in the horizontal canal is critical, and thorough knowledge of skull base anatomy in the area is necessary to avoid damage to the cochlea while optimizing the length of artery exposed for performing the bypass. The greater superficial petrosal nerve is divided to avoid traction on the facial nerve. Care must be taken not to damage the adjacent tensor tympani muscle and the eustachian tube.

The cervical ICA is exposed through a separate neck

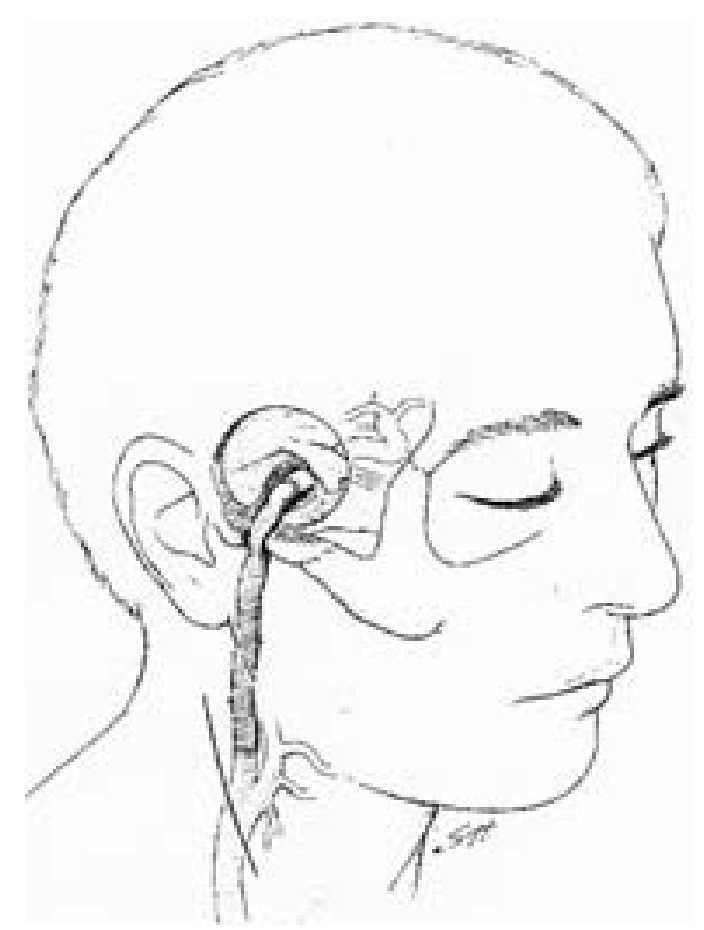

Fig. 1. Artist's drawing demonstrating the cervical-to-petrous ICA bypass. Used with permission from Fitzpatrick, et al. incision. The SVG is harvested from the groin or leg. Surgical nuances in SVG extraction and preparation have been well described by Sundt and Sundt. ${ }^{20}$ The proximal end of the saphenous vein is anastomosed end-to-end to a stump of the cervical ICA. The vein graft is then tunneled either via a subcutaneous or a submandibular route. The SVG can be passed inside a disposable chest trocar tube that is introduced in the cervical area through a route deep to the mandible, temporalis muscle, and zygoma toward the subtemporal petrous ICA via the pterygoid zygomatic fossa. ${ }^{1,8}$ Alternatively, the graft can be tunneled subcutaneously and laid in a groove drilled into the zygoma and lateral floor of the temporal fossa. ${ }^{3}$ Sekhar and Kalavakonda ${ }^{13}$ have described both pre- and postauricular subcutaneous tunnel techniques for routing of the graft. Of these, the preauricular tunnel is preferred because it allows the graft length to be shorter and assume a more physiological orientation.

The distal end of the SVG is then anastomosed end-toside or end-to-end to the petrous CA. Connection of the graft to the horizontal petrous CA remains one of the most challenging exercises in skull base and cerebrovascular surgery. This is because the working space required to perform the surgical connection to a small segment of the recipient vessel is both deep and restricted. If performing an end-to-side anastomosis, after applying vascular clips proximal and distal to the desired segment for the distal anastomosis (alternatively, the proximal side may be ligated using suture to provide more room), a small arteriotomy is made in the horizontal petrous ICA. The SVG is anastomosed with interrupted No. 8-0 monofilament nylon suture. The vascular clip on the distal ICA is temporarily removed to flush the graft and to assess the anastomotic site. If an end-to-end anastomosis is performed, the proximal aspect of the donor segment is ligated and the artery is sharply transected prior to anastomosis. After anastomosis, the temporary clips are removed and the anastomotic sites are inspected for patency.

\section{Petrous-to-Supraclinoid ICA (Fukushima) Bypass}

The petrous-to-supraclinoid ICA bypass is an effective revascularization strategy for lesions of the cavernous sinus when the intracavernous ICA must be sacrificed (Fig. 2 ). Giant aneurysms of the cavernous CA not amenable to clip therapy (Fig. 3), as well as tumors encasing the cavernous ICA in cases involving preexisting cranial neuropathies in which an oncological resection is planned, may require a cerebrovascular bypass. In 1986 Fukushima performed a petrous-to-supraclinoid (C5-3) ICA interpositional saphenous vein bypass to trap a giant cavernous aneurysm (unpublished data). Spetzler, et al., ${ }^{17}$ subsequently reported the use of this technique in 18 patients for the management of intracavernous giant aneurysms, tumors, and CA stenosis. Sekhar, et al., ${ }^{16}$ performed the same bypass technique to treat four patients with intracavernous neoplasms invading the ICA and two patients with cavernous CA aneurysms.

The Fukushima bypass technique has several distinct advantages. It establishes a high-flow venous graft that is entirely intracranial, which avoids stress on the graft associated with head and neck movements. The relatively short length of the bypass also reduces the increased risk 

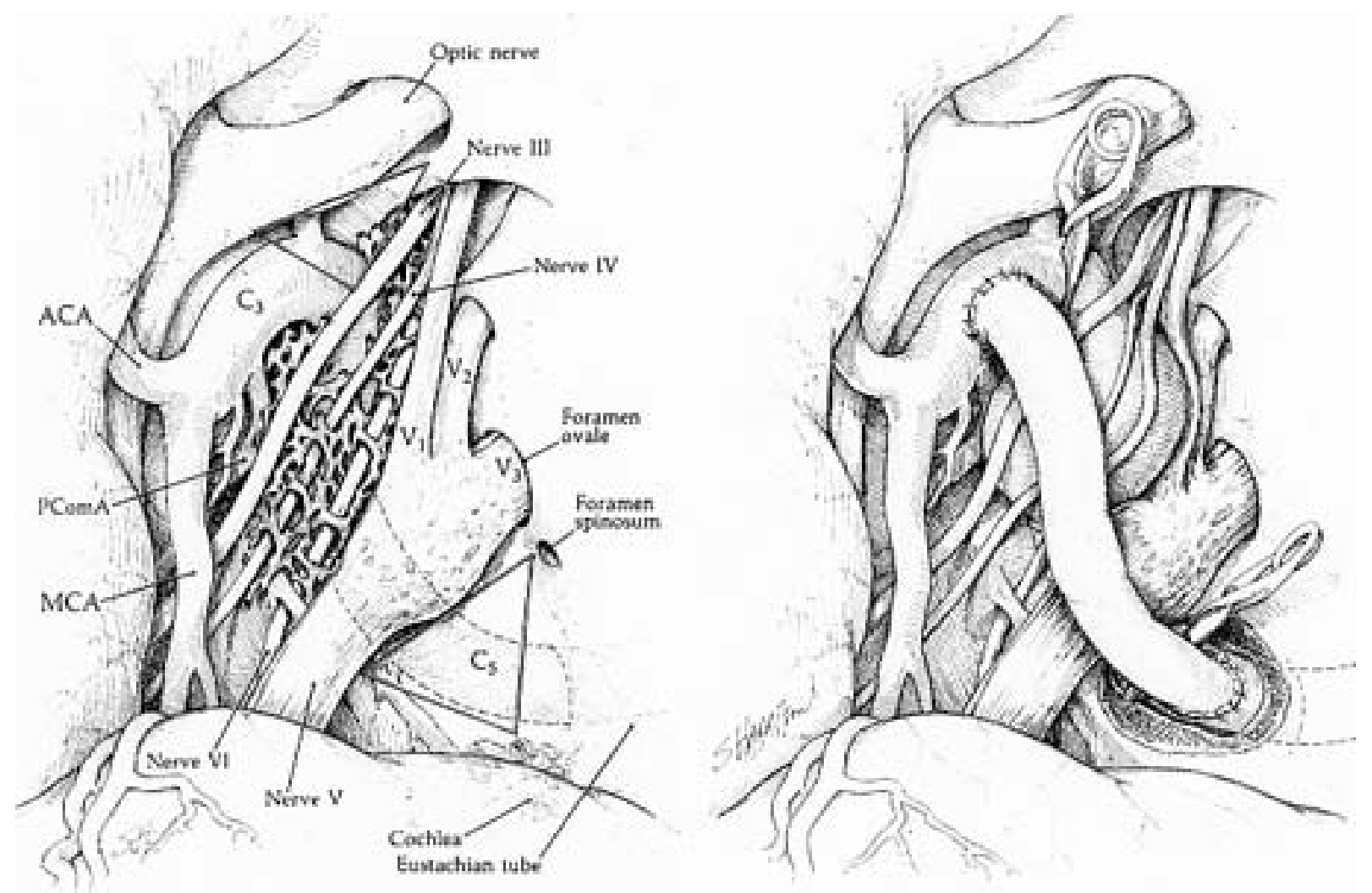

Fig. 2. Schematic illustrations. Left: The surgical anatomy and exposure for the petrous-to-supraclinoid (C5-3) ICA bypass. $\mathrm{ACA}=$ anterior cerebral artery; $\mathrm{PcomA}=$ posterior communicating artery; $\mathrm{MCA}=$ middle cerebral artery. Right: The petrous-to-supraclinoid (C5-3) ICA bypass. Used with permission from Spetzler, et al.

of thrombosis associated with longer grafts. Trapping the cavernous CA by placing clips immediately adjacent to the proximal and distal ends of the bypass eliminates vascular dead space that might otherwise encourage thrombus formation leading to delayed complications secondary to extension of clot. The disadvantages of this approach are the aforementioned technically challenging, small working space during the proximal anastomosis to the petrous CA, with an associated relatively prolonged temporary occlusion time of the ICA.

A standard frontotemporal craniotomy is performed on the side of the lesion. The sphenoid ridge, anterior clinoid process, and proximal optic canal are removed using a high-speed drill. The sylvian fissure is then opened. Division of the fibrous dural ring at the intra- and extradural junction surrounding the ICA, as described by Dolenc, ${ }^{2}$ is performed to provide optimal exposure for clip placement on the ICA, proximal to the ophthalmic artery takeoff. The segment between the ophthalmic and posterior communicating arteries is used for the distal anastomosis of the SVG.

Exposure of the C-5 portion of the CA is performed extradurally. ${ }^{5}$ The dura of the temporal lobe is carefully separated from the temporal fossa and elevated along the course of the middle meningeal artery to the foramen spinosum. The middle meningeal artery is coagulated and divided, and exposure is continued medially and anteriorly to the foramen ovale. Using a high-speed drill, the pe- trous portion of the ICA is exposed just posterior to the foramen ovale and medial to the foramen spinosum. Approximately $1 \mathrm{~cm}$ of the petrous CA is exposed for the bypass. The eustachian tube, which runs just lateral to C-5, can be left intact.

An SVG is harvested from the thigh. Propofol or shortacting barbiturate is administered to induce electroencephalographic burst suppression before ICA occlusion. Burst suppression is continued until after the SVG anastomosis and reperfusion is confirmed using micro-Doppler ultrasonography. A temporary proximal clip (or, alternatively, suture ligation) and a distal permanent clip are applied to the horizontal segment of the petrous ICA. An arteriotomy is made and the SVG is anastomosed end-toside to C-5 by using interrupted No. 8-0 monofilament nylon sutures. Alternatively, an end-to-end anastomosis is performed particularly in cases in which an oncological resection of cavernous sinus tumor encasing the ICA is planned.

The distal portion of the SVG is anastomosed end-toside to the supraclinoid ICA between the ophthalmic and posterior communicating arteries. This is performed using No. 8-0 or 9-0 suture. Vascular clips are permanently left distal to the anastomosis on the petrous CA (if not suture ligated) and proximal to the ophthalmic artery takeoff.

\section{Cervical-to-Supraclinoid ICA Bypass}

This is the most common skull base bypass performed 


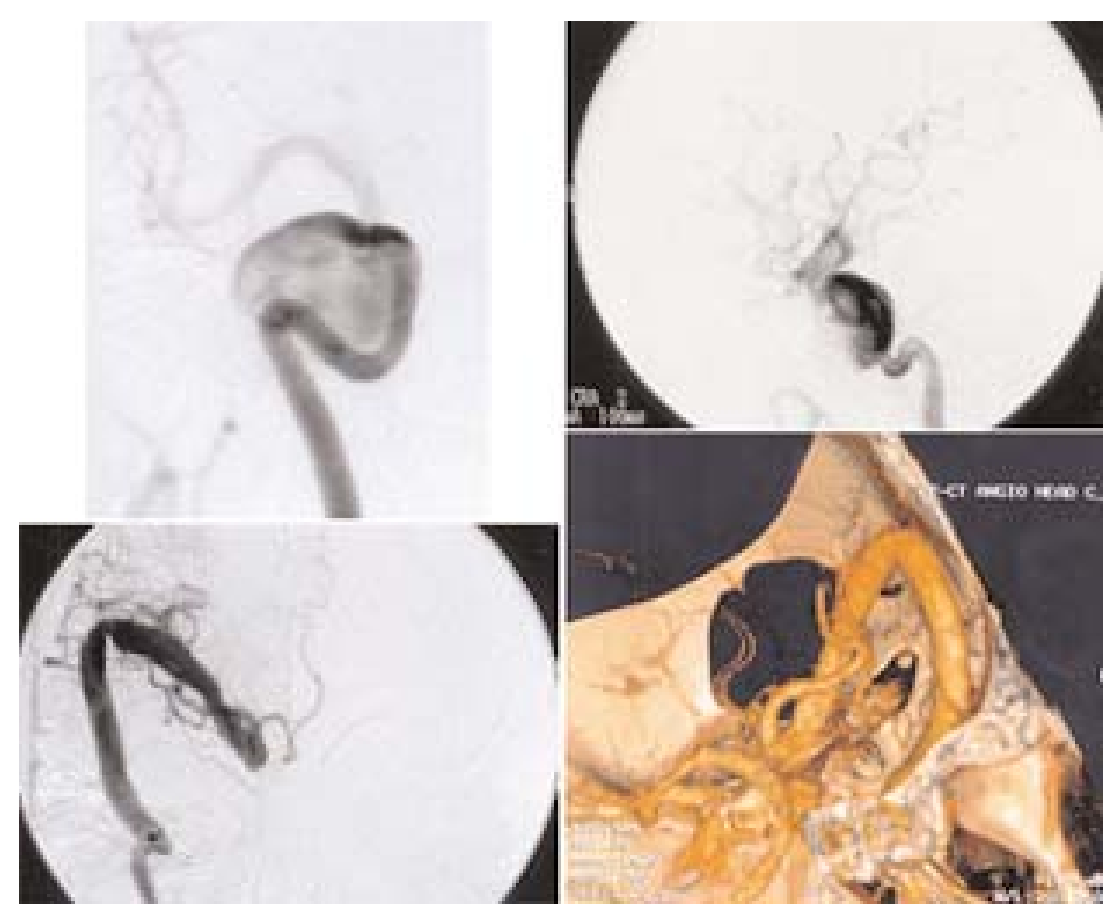

Fig. 3. Angiograms and CT angiogram obtained in a 62-year-old woman with a large intracavernous CA aneurysm treated with a petrous-to-supraclinoid interposition saphenous vein bypass. Upper Left and Right: Preoperative anteroposterior (upper left) and lateral (upper right) right-sided ICA angiograms demonstrating a large intracavernous CA aneurysm. Lower Left and Right: Postoperative (lower left) anteroposterior angiogram and CT angiogram (lower right) demonstrating graft patency.

in our institution. Although cervical ICA-to-MCA interposition techniques have been used by others in the past, the senior author (W.T.C.) prefers to perform a high cervical-to-supraclinoid ICA bypass that is routed in the sub-

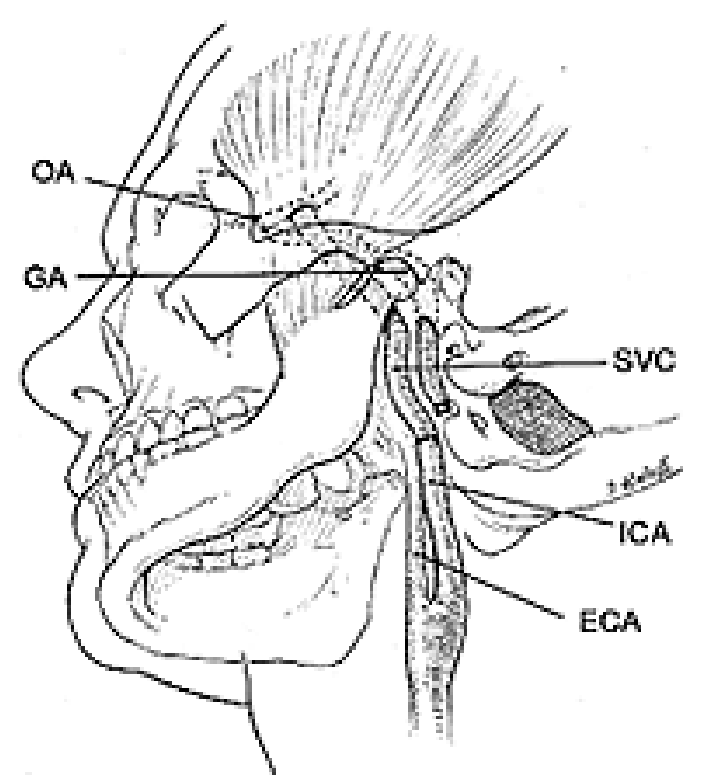

Fig. 4. Artist's illustration demonstrating the cervical-to-supraclinoid submandibular saphenous vein bypass. GA = giant petrous aneurysm (excluded); ECA = external carotid artery. Used with permission from Couldwell, et al. mandibular space to provide high anterograde flow with a short interposition conduit whenever possible. ${ }^{1}$ A standard pterional craniotomy is performed on the side of the lesion. The lateral aspect of the sphenoid wing and the anterior clinoid process are removed extradurally by using a high-speed drill. This allows adequate exposure to the ophthalmic segment of the ICA, which serves as the recipient vessel for the saphenous vein bypass. A neck incision is made along the anterior border of the sternocleidomastoid muscle, and the cervical ICA is isolated. A segment of approximately $20 \mathrm{~cm}$ of the greater saphenous vein is harvested and perfused with heparinized saline.

In performing this particular bypass, we prefer to tunnel the graft via the submandibular route (Figs. 4 and 5). ${ }^{1}$ One advantage is that it permits a more direct routing of the bypass graft to the recipient ophthalmic segment of the ICA. When the graft is tunneled superiorly and cut to the proper length, the overall length of the graft is shortened, thereby promoting graft patency. In addition, the submandibular placement of the graft provides physical protection of the graft under the mandible, temporal muscle, and zygoma.

Prior to making the submandibular pass, the zygoma is removed and reflected inferiorly with the masseter and temporalis muscles. A bone trough is also created at the middle fossa skull base to provide room for the graft. This step is important in avoiding graft compromise by mandibular movement. After induction of burst suppression with intravenous propofol or barbiturate and administration of $5000 \mathrm{U}$ of intravenous heparin, the cervical ICA is ligated as high in the neck as possible (this will ultimate- 


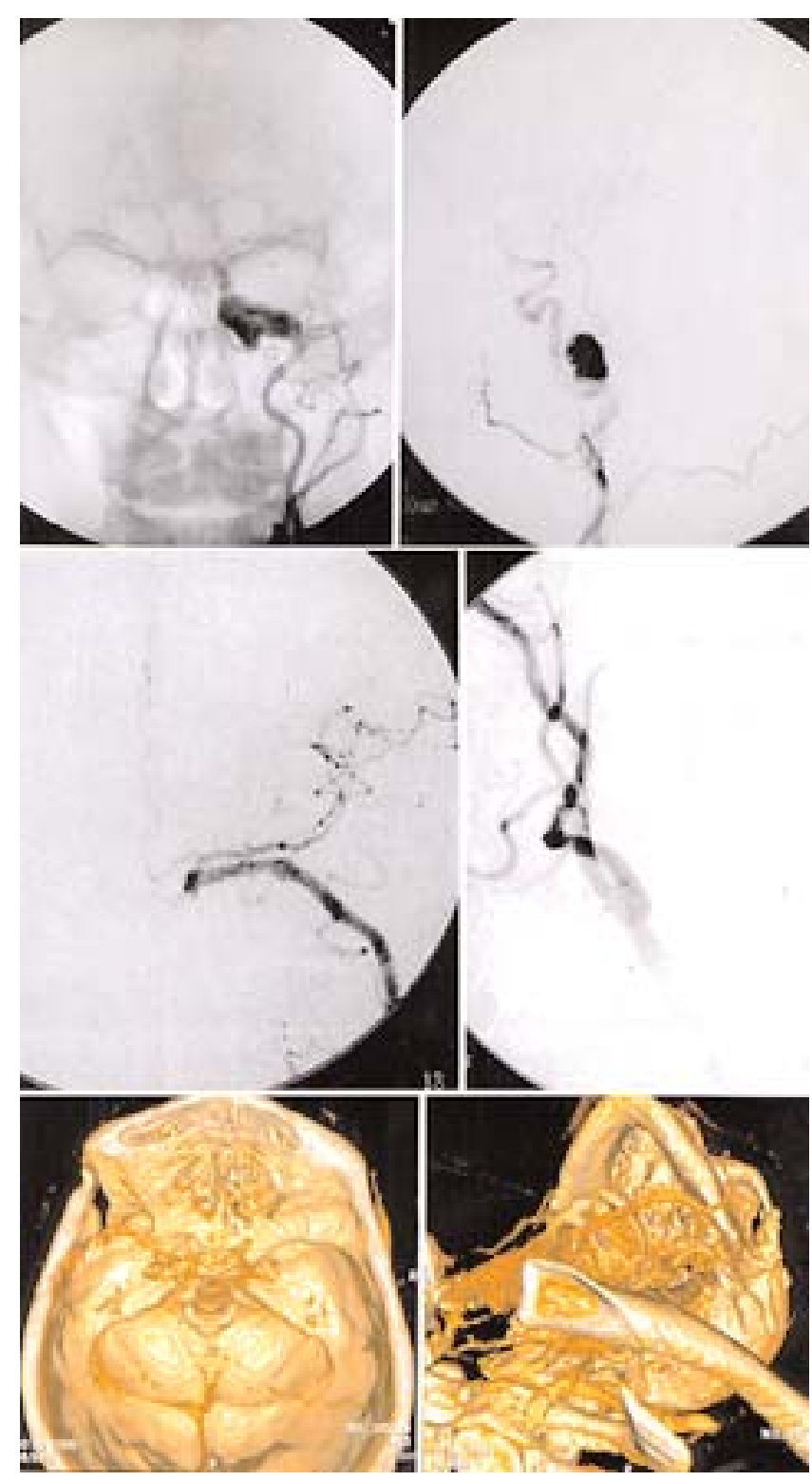

Fig. 5. Angiograms and CT angiogram obtained in a 47-yearold man with a giant petrous CA aneurysm treated with a cervicalto-supraclinoid submandibular saphenous vein bypass. Upper Left and Right: Preoperative angiograms demonstrating the giant petrous CA aneurysm. Center Left and Right: Postoperative angiograms demonstrating the patency of the graft and the exclusion of the giant aneurysm from the circulation. Lower Left and Right: Computerized tomography angiograms demonstrating routing of the graft and patency during mandibular movement. Used with permission from Couldwell, et al.

ly shorten the graft length). An end-to-end proximal anastomosis is performed using the SVG. The ICA may be temporarily mobilized inferiorly, which enables an easier technical proximal anastomosis. By using a short ventriculoperitoneal shunt passer or large cannula to accept the graft, the distal graft is advanced beneath the mandible and through the skull base without causing trauma. The graft is sized appropriately and surgically attached to the ophthalmic segment of the ICA between the ophthalmic artery and posterior communicating artery takeoffs. This is usually performed end-to-side to preserve the ophthalmic takeoff, or, if the OA is not preserved, the anastomosis is performed end-to-end. A permanent vascular clip is placed, or suture ligation is performed just proximal to the OA branch. This particular bypass technique has proved to be useful, to be associated with excellent long-term patency rates, and is an effective alternative route for interpositional vein graft placement in cases in which highflow revascularization is desired.

\section{CONCLUSIONS}

Cerebral revascularization may be necessary when the surgical treatment of complex aneurysms and skull base tumors requires sacrifice of the ICA. The cervical-topetrous, petrous-to-supraclinoid, and cervical-to-supraclinoid CA high-flow saphenous vein bypass procedures are important interventions in the armamentarium of the cerebrovascular or skull base surgeon.

\section{References}

1. Couldwell WT, Zuback J, Onios E, et al: Giant petrous carotid aneurysm treated by submandibular carotid-saphenous vein bypass. Case report. J Neurosurg 94:806-810, 2001

2. Dolenc VV: A combined epi- and subdural direct approach to carotid-ophthalmic artery aneurysms. J Neurosurg 62: 667-672, 1985

3. Fitzpatrick BC, Spetzler RF, Ballard JL, et al: Cervical-topetrous internal carotid artery bypass procedure. Technical note. J Neurosurg 79:138-141, 1993

4. Gelber BR, Sundt TM Jr: Treatment of intracavernous and giant carotid aneurysms by combined internal carotid ligation and extra- to intracranial bypass. J Neurosurg 52:1-10, 1980

5. Glasscock ME: Exposure of the intra-petrous portion of the carotid artery, in Hamberger CA, Wersall J (eds): Disorders of the Skull Base Region: Proceedings of the Tenth Nobel Symposium, Stockholm, 1968. Stockholm: Almqvist \& Wiksell, 1969, pp 135-143

6. Lawton MT, Hamilton MG, Morcos JJ, et al: Revascularization and aneurysm surgery: current techniques, indications, and outcome. Neurosurgery 38:83-94, 1996

7. Linskey ME, Jungreis CA, Yonas H, et al: Stroke risk after abrupt internal carotid artery sacrifice: accuracy of preoperative assessment with balloon test occlusion and stable xenon-enhanced CT. AJNR 15:829-843, 1994

8. Miyazaki S, Fukushima T, Fujimaki T: Resection of high-cervical paraganglioma with cervical-to-petrous internal carotid artery saphenous vein bypass. Report of two cases. J Neurosurg 73:141-146, 1990

9. Roski RA, Spetzler RF, Nulsen FE: Late complications of carotid ligation in the treatment of intracranial aneurysms. J Neurosurg 54:583-587, 1981

10. Sekhar LN, Bucur SD, Bank WO, et al: Venous and arterial bypass grafts for difficult tumors, aneurysms, and occlusive vascular lesions: evolution of surgical treatment and improved graft results. Neurosurgery 44:1207-1224, 1999

11. Sekhar LN, Chandler JP, Alyono D: Saphenous vein graft reconstruction of an unclippable giant basilar artery aneurysm performed with the patient under deep hypothermic circulatory arrest: technical case report. Neurosurgery 42:667-673, 1998

12. Sekhar LN, Duff JM, Kalavakonda C, et al: Cerebral revascularization using radial artery grafts for the treatment of complex intracranial aneurysms: techniques and outcomes for $17 \mathrm{pa}-$ tients. Neurosurgery 49:646-659, 2001 
13. Sekhar LN, Kalavakonda C: Cerebral revascularization for aneurysms and tumors. Neurosurgery 50:321-331, 2002

14. Sekhar LN, Kalavakonda C: Saphenous vein and radial artery grafts in the management of skull base tumors and aneurysms. Oper Tech Neurosurg 2:129-141, 1999

15. Sekhar LN, Patel SJ: Permanent occlusion of the internal carotid artery during skull-base and vascular surgery: is it really safe? Am J Otol 14:421-422, 1993

16. Sekhar LN, Sen CN, Jho HD: Saphenous vein graft bypass of the cavernous internal carotid artery. J Neurosurg 72:35-41, 1990

17. Spetzler RF, Fukushima T, Martin N, et al: Petrous carotid-tointradural carotid saphenous vein graft for intracavernous giant aneurysm, tumor, and occlusive cerebrovascular disease. J Neurosurg 73:496-501, 1990

18. Sundt TM Jr, Piepgras DG, Houser OW, et al: Interposition saphenous vein grafts for advanced occlusive disease and large aneurysms in the posterior circulation. J Neurosurg 56: 205-215, 1982

19. Sundt TM Jr, Piepgras DG, Marsh WR, et al: Saphenous vein bypass grafts for giant aneurysms and intracranial occlusive disease. J Neurosurg 65:439-450, 1986

20. Sundt TM III, Sundt TM Jr: Principles of preparation of vein bypass grafts to maximize patency. J Neurosurg 66:172-180, 1987

Manuscript received January 15, 2003.

Accepted in final form February 7, 2003.

Address reprint requests to: William T. Couldwell, M.D, Ph.D., Department of Neurosurgery, University of Utah, 30 North 1900 East, Suite 3B409, Salt Lake City, Utah 84132. email: william.couldwell@hsc.utah.edu. 